\title{
Colloids and colloidal systems in human health and nutrition
}

\begin{abstract}
Colloids and colloidal systems are essential to life. They are extremely useful, even indispensable, in many commercial and industrial situations as well. They function in everybody cell, in the blood, and in all body fluids, especially the intercellular fluids, formerly known as "humours." Therefore, increased understanding of colloids and their attendant phenomena, as well as the application of their operating principles, to enhance human health considerably is discussed in this article. Colloidal science is relatively young, however, and the number of qualified experts is few compared to other areas of science. In addition, most study in colloids has been confined to industrial processes. Having found use in agriculture, the concrete industry, horticulture, the floral industry, mining, electroplating and cosmetics, to name a few, colloids may have specific application in almost every form of human endeavour. Each of the few general areas just mentioned can be broken down into extensive subdivisions as discussed in this article.
\end{abstract}

Volume 3 Issue 6 - 2016

\author{
Robert OYoung \\ Universal Medical Imaging Group, USA
}

Correspondence: Robert O Young, pH Miracle Inc., 16390 Dia del Sol,Valley Center, California, 92082, USA, Tel 760 75I 832I, Email universalmedicalimaging@yahoo.com

Received: January 03, 2016 | Published: May 27, 2016

Keywords: colloids, colloidal chemistry, colloidal silver, health, nutritional supplements

\section{General information}

\section{Definition of terms}

A material system in which one kind of matter, usually in the form of fine particles, is distributed more or less evenly throughout another is called dispersion. The term colloid is usually applied either to a particle of matter falling within a specified size range, or to a colloidal system, which is a combination of particles and a containing medium, i.e., a dispersion. In this essay, colloid will refer to particles only and colloidal system to the dispersion. A colloidal system may consist of one kind of colloid or a combination of solid, liquid or gas colloids dispersed in the medium. Essentially, particle size distinguishes colloidal systems from other material systems, such as suspensions and solutions (suspensions have larger particles and solutions have smaller).

\section{Classification of dispersions}

In 1925, H Freundlich classified dispersions into three basic categories: true solutions, colloidal solutions, and emulsions/ suspensions. There were four parameters for categorizing dispersion: (1) particle size; (2) Brownian movement (movement of suspended particles resulting from the impact of molecules of the medium); (3) ability to pass through ordinary filter paper; and (4) level of solubility. Freundlich's success in categorizing dispersions was somewhat limited due to the laboratory equipment available at that time. As a result, he and his contemporaries had to calculate the size of the particles. The table below identifies these categories of solutions (Figure 1).

Half a century later, the Russian scientist SS Voyutsky wrote his book on colloidal chemistry, and his findings. ${ }^{1}$

Particle size was still of major importance in distinguishing systems from one another, but although the range of particle sizes in the diagram is the same as that originally calculated, the difference is that the emulsion/suspension level is divided into the micro heterogeneous level (next up from colloidal) and the coarsely dispersed system. Voyutsky's term for a true solution is the more accurate molecularly dispersed system, in which particles tend to be below 1 nanometer $(\mathrm{nm})$. Though particle size is critical in making distinctions among systems, exact points of transition between the various degrees of dispersion cannot be established. Rather, there is continuity much like the colour transitions in a rainbow. Even so, colloidal systems are quite different from micro-heterogeneous ones, which do not have the very large total surface area of colloids.

\begin{tabular}{|c|c|c|c|}
\hline Property & Suspension & Colloidal sol. & Solution \\
\hline Particle size & $>100 \mathrm{~nm}$ & 1 to $100 \mathrm{~nm}$ & $<100 \mathrm{~nm}$ \\
\hline $\begin{array}{l}\text { Filtration } \\
\text { separation }\end{array}$ & Possible & Not possible & Not possible \\
\hline Settling of particles & Settle on their own & $\begin{array}{l}\text { Settle on } \\
\text { centrifugation }\end{array}$ & Do not settle \\
\hline Appearance & Opaque & Transparent & Transparent \\
\hline Tyndall effect & Shows & Shows & Does not show \\
\hline $\begin{array}{l}\text { Diffusion of } \\
\text { particles }\end{array}$ & Do not diffuse & Diffuse slowly & Diffuse rapidly \\
\hline $\begin{array}{l}\text { Brownian } \\
\text { movement }\end{array}$ & May show & Shows & $\begin{array}{l}\text { May or may not } \\
\text { show }\end{array}$ \\
\hline Nature & Heterogeneous & Heterogeneous & Homogeneous \\
\hline
\end{tabular}

Figure I The differences between suspensions, solutions and colloidal solutions.

In certain concentrations, micro heterogeneous systems display many of the same characteristics as colloids because a lot of the particles are within the colloidal range. However, due to the fact that a critical percentage of the particles are larger than $100 \mathrm{~nm}$, a significant number of them will tend to settle. Micro-heterogeneous systems are a different colour than a corresponding colloidal system. The color will tend more toward black because light is blocked by the coarser particles, and this will increase as concentration is raised. (See "Testing a Colloidal System" below.) Colloids may also be found in micro heterogeneous systems and coarse suspensions. Relatively speaking, the total area of coarsely dispersed systems is quite small.

\section{Colloidal characteristics}

Colloid size ranges from 0.001 to 0.1 micron ( 1 to $100 \mathrm{~nm}$ ) in diameter. Since a micron is one-millionth of a meter, and a meter is about 40 inches, a micron is four one-hundred- thousandths of an 
inch. Thus, a colloid measures about four-millionths of an inch to about four one-hundred-millionths of an inch, or 10 angstroms at the smaller end of the range. This puts the size of the smallest colloids at about 10 times the size of a hydrogen atom.

Colloids do not settle, and are fdterable by ordinary techniques in the same sense as fdterable bacteria, whereas coarser particles in the dispersion size range are retained. They differ from "particles" in molecularly dispersed systems in that dispersed colloids cannot pass through the fine pores of passive membranes. Because of their size, colloids diffuse slowly.

In addition to particle size, a colloidal system must have the following three properties in order to be differentiated from other dispersions:

1. It must be heterogeneous, i.e., consist of dissimilar constituents, for example silver and water.

2. It must be multiphasic, i.e., solid/liquid, gas/liquid, etc.

3. The particles must be insoluble in the solution or suspension.

Each of these characteristics interacts with the others, giving a colloidal system its unique qualities. One fascinating thing is that even though particle size and concentration may vary, as long as most particles are in the proper range, the system will retain its colloidal properties, even though it may not be ideal.

Colloidal systems may be further identified according to the phases of their constituents. A solid dispersed in a liquid is called a sol, a solid or semi-solid colloidal system is a gel. An emulsion consists of one liquid dispersed in another. An aerosol, such as smoke or mist, consists of a solid or liquid dispersed in a gas. Some alloys are solidin-solid colloids. The most common system, especially where human physiology is concerned, is the sol, or solid-in-liquid dispersion. ${ }^{2-7}$

\section{Sol interaction}

There are three main classifications of sols based upon the interaction of the particles and the medium:

1. Lyophobic (solvent-hating);

2. Lyophilic (solvent-loving); and

\section{Association colloids.}

Lyophobic colloids have little attraction for the medium and are therefore not highly stable (not able to remain dispersed). Metallic sols in water are examples of lyophobic colloids, that is, they lack the affinity for that medium. But they are atypical lyophobics due to the exceptional ability of metals to hold a charge. In lyophilic systems the particles are attracted to the medium. These systems are inherently more stable than lyophobic ones because the attraction of the colloid for the medium resists settling. Both types of colloidal systems are found in plant and animal fluids.

The molecules of association colloids have a hydrocarbon chain with a hydrophilic "head" group and a hydrophobic "tail" group. When dissolved in water the molecules form clusters called micelles. These keep the head groups in contact with the water while protecting the tails within the micelle from water. The interior of the micelle corrals oily material and keeps it stable within the system. Detergents and soaps are common examples of association colloids.

\section{Surface charge}

The most important characteristic of colloidal systems is surface charge on the particles. Keep in mind that a "particle" is a group of bonded atoms or molecules. Charged particles repel each other, overcoming the tendency to aggregate (the attraction force) and remaining dispersed. Particle size plays a major role in the capacity to bear a charge, and the colloidal size range is set by this capacity. In manufactured systems, the charge can be greatly increased over what might occur naturally. Within the range, the smaller the particle, the greater the surface and the greater the charge that can be applied in manufacture. Only heterogeneous, highly dispersed colloidal systems, containing the smallest possible particles, have a well-developed surface area. Given a constant voltage applied to the system, particle charge is not automatically increased as the substance is made finer, but total charge in the system will increase.

Already coarse particles will tend to fall out even if they have received an electrical charge like the smaller particles, because gravity will have a greater influence than the electrical forces which maintain the dispersion. Metallic particles have a great affinity for each other at the atomic level. They are magnetically attracted to each other and want to bond. But the magnetism of metals does not create an added difficulty of attraction against maintaining a colloidal system because of the superior capacity of metals to hold a charge.

Given a constant particle size, the higher the concentration in a solution, the more likely the attraction force will overcome the repelling charge, creating larger masses. At some point, the mass will precipitate out due to gravitation. At lesser concentrations, the attraction force is insufficient for precipitate particle bonding, and groups are light enough that gravitation will not pull them out of solution. This is an ideal colloidal system.

\section{Manufacturing colloids and systems}

At least five methods were used to manufacture colloids before 1938, including: (1) Grind, (2) Wave, (3) Liquid, (4) Chemical, (5) Electrical. For medical or health purposes, the FDA now allows both the grind and electrical manufacturing techniques to be used. Of these two methods, however, the electro-colloidal process is generally considered to be far superior. (The chemical method, described below, is restricted to industrial or commercial applications.) With the grind method, the inorganic or organic particles are usually no finer than four one-hundred-thousandths of an inch, or about one micron, which is outside the upper end of the ideal size range by a factor of 10. Such particles may or may not be electrically charged. Even if a charge is present, the size of the particles may be great enough that the repelling forces are unable to overcome the pull of gravity. Thus, particles will tend to settle to the bottom of the solution, and much of the effectiveness of the colloidal system will be lost. While some sols owe their stability to particle size, charge and high dispersion, others employ a mechanical stabilizer added to the medium. Such stabilizers include gelatine, glycoprotein's, and starch, among other things, which increase solution viscosity and cause the particles to settle much more slowly. The downside to this is that stabilizers tend to block the effects of the colloids, and the particles will still eventually settle if the solution is allowed to stand long enough. If the inorganic or organic particles are within the size range of 1 to 100 $\mathrm{nm}$ and are uniformly charged, no stabilizer is required to maintain suspension indefinitely in deionised water, as long as no disruptive influence intrudes. Thus, the integrity and power of a colloidal system is a factor of the interplay among size, charge, concentration, and interaction between particle and medium. It should be mentioned that shape is also a factor.

In recent years, the chemical process has been widely employed to replace the inferior grind method, because it provides a convenient shortcut to the more difficult electro- colloidal process. But it also 
has drawbacks, one of which is the difficulty in getting the chemicals (acids) back out of the colloidal solution. Consequently, traces of the chemicals are frequently left in solution, which can cause unwanted effects, especially in nutritional/medical applications. After studying the health benefits of various forms of colloidal silver, Dr. Leonard Keene Hirschberg, A.M.M.D. (Johns Hopkins) concluded, "There are two principal ways of producing metallic colloids, viz., chemical and physical (electrical). The two methods yield widely different results, and from a therapeutic point of view I need only deal with the electric colloid metals, since only these present the necessary homogeneity, minuteness of granules, purity, and stability. ${ }^{7-15}$ "

A simple illustration will suggest the immense power potential of a colloidal system. The total surface of a one-inch cube of iron is six square inches. By colloidal chemistry, the cube can be divided into particles having a total surface area in the range of $800,000,000$ square inches, all expressing electrical energy. The total surface area of the particles in a quarter teaspoon is greater than that of a football field.

\section{The ultimate colloid}

The highest quality colloidal systems are produced by the electrocolloidal method, meaning the inorganic or organic particles and (usually) water have been completely "collided." This is simultaneous dispersion and bonding by a current sent through the combination. This is the only method that will create a true colloidal system by manufacture. Products that are simple mixtures of metal and liquid cannot possess nearly the potential of electro colloids, and are therefore of questionable value. The proper electrical process allows inorganic or organic particles that are well within the colloidal size range to be drawn off an ingot. Animated by Brownian movement, they are able to remain in suspension in a liquid medium almost indefinitely. (Because many nutrients are best transported through the body in water, the best medium to use for ingested nutritional products is pure, de-ionized water).

All other things being equal, the number of particles varies inversely according to the cube of the size change, so if size is reduced 50 percent, overall number is multiplied by eight. This is a mathematical proof, and is determined by actual count using an electron microscope and by atomic absorption.

Obviously, ideal size is element dependent. Size is controlled by frequency, amperage and micro-meshes, among other things.

The ultimate colloidal sol contains ultra-fine and ultra-light particles in the range of 0.015-0.005 microns in diameter, and they will remain suspended in de-ionized water without need of any other ingredient. There is no visible accumulation of inorganic or organic particles either in the solution or settled on the bottom. Products that show visible particles in the solution or at the bottom of the container indicate that the particles are either too large or have not received the proper electrical charge.

The metallic particles in a sol may vary in concentration, but more is not necessarily better, unless we have correspondingly smaller particles. In fact, the reverse is usually true- less is better, and in essence, less is more, functionally speaking, because as noted earlier, the higher the concentration in a solution, the more likely the attraction force will overcome the repelling charge. But even before this happens, effectiveness is reduced. The highest quality colloid will have a certain maximum number of particles. They will be of the minimum possible size, and ideally no more than a "handful" of atoms hooked together per molecule of water utilized, and in a negatively charged state. This will prevent further aggregation at that size.

\section{Testing a colloidal system}

A quick way to see if a solution contains colloids is by observing the Tyndall effect. A clear colloidal dispersion will appear turbid when a sharp and intense beam of light is passed through. The scattered light also takes on a cone shape within the solution. A simple way to observe this is to shine a very bright flashlight through a test tube of the dispersion in a dark room (Figure 2).

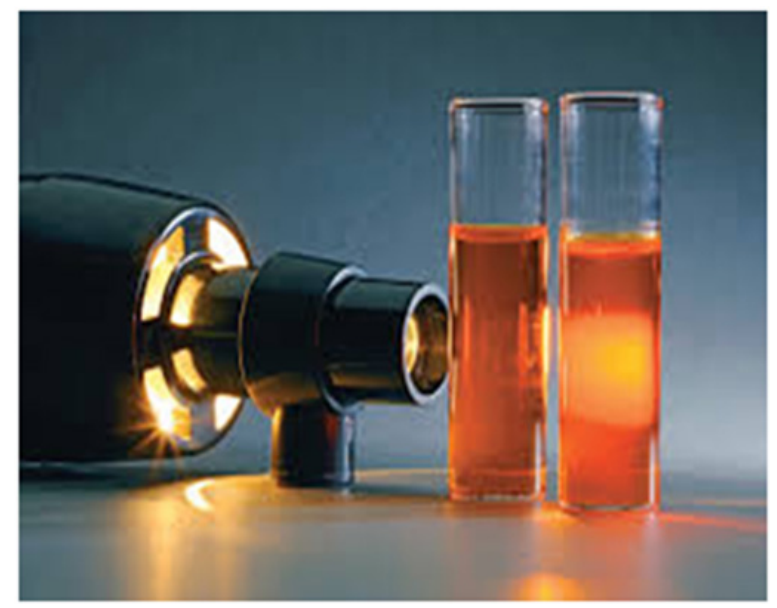

Figure 2 Viewing the colloidal particle size of silver using a laser beam.

As noted earlier, a critical indicator of a colloidal system's quality is its color. The ideal form of colloidal silver, for example, will have a golden yellow color. As the size of each particle increases, the color of the suspension proceeds from yellow to brown to red to gray to black. Therefore, the color range could also be read as "best to fair to mediocre to inferior." In all cases, systems produced using the electro-colloidal methods are a different color than those from other methods except where an artificial dye is used to imitate the proper color. Additionally, color varies with concentration, use of a stabilizer, and the presence or absence of other trace elements.

To confirm that a product is a true colloidal, examine the ingredients. If it contains an ingredient other than the designated colloidal particles, the product may not be suitable. If no additional ingredient is listed, but the product requires refrigeration, it means there is an ingredient in it that might spoil at room temperature. Properly prepared using the electro-colloidal method, a colloidal system requires no such ingredient. Needless to say, a product with instructions to shake before using is also quite suspect.

\section{Colloids and the living system}

Humeral medicine died in 1863 as a result of the influence of the Virchow school. Cellular pathology tells only part of the story of disease. The colloidal integrity of the intercellular fluids, or humeral milieu, is the sine qua non of life itself. The state of health is present when there is unhindered flow of life- force through every part of the humeral system. The real function of body cells is to maintain the milieu in a state of functional efficiency -OC Gruner.

\section{Colloid pioneers}

At the present time, colloidal chemistry plays a major role in over 7,000 industries, and, as noted earlier, most study in colloids has been applied to industrial processes. In the last few decades of the 19th century, and even well into the 20th, there was considerable awareness of the importance of colloids to human health. But with the rise and sway of monomorphic, allopathic, cell-oriented medicine, this area of study fell by the wayside. Therefore, there is currently comparatively 
little awareness of, or focus on, colloids in the living system. Professor Wolfgang Ostwald has noted, "All life processes take place in a colloidal system, and that is true both of the normal fluids and secretions of the organism, and of the bacterial toxins, as well as, in large measure, of the reactions which confer immunity." Based on that premise, Alfred Searle wrote: Fortunately, the recognition of bacteria and their products as essentially colloidal in character has greatly facilitated the study of disinfection. It is now realized thatdisregarding the fact that bacteria are alive-they may, owing to their colloidal character and that of the toxins and some other substances they produce-be destroyed by substances which bear an electrical charge opposite to that of the bacteria or their colloidal products. "The great advantage of dealing with germs as colloids lies in the fact that the agents used for their coagulation and consequent destruction is not necessarily poisonous."

Colloidal silver, for example, has no recorded side effects. Its power to "disinfect" stems from the frequencies of vibration presented on the surface of the particles; i.e., they are very highly charged oppositely to microorganisms, and very active. Yet they are completely safe and natural. It is a situation opposite to the antibiotic one, which is "toxichemical," not only to the target microform but to the host as well, and which sets more improperly charged toxins free in the system rather than catalytic, energetically harmonious colloids. Moreover, the antibiotic cannot really alter the condition which supported the evolution of the target form, and may not even kill it but only instigate an evasive pleomorphosis and potentiate a worse scenario later. This is not to mention the negative effect on the health and vitality of the intestinal villi. (The intestinal villi are safe from colloidal silver because the silver is absorbed and/or used to buffer dietary acids in the mouth and stomach before reaching the small intestines and the intestinal villi).

\section{Alfred searle}

Metal solids have the further therapeutic advantage of acting most rapidly in faintly alkaline solutions, so that when properly prepared they are not affected adversely by normal blood. Before a therapeutic substance can exert its full effect it must be converted into the ionized or into the colloidal state. ... The drugs employed to combat disease should be in the colloidal state, i.e. in a form in which they may be isomorphic and isotonic with the elements of the body. Only so can they be expected to exert their full potency.

The task of thus bringing their remedial virtue to its highest point is not an easy one, for colloidal substances, unless prepared with consummate skill and meticulous care, lack stability, and are prone to precipitation when brought into contact with the electrolytes normally present in the body tissues and fluids.

Though Searle was speaking in terms of pharmaceuticals, the information also applies to food supplements.

\section{Colloidal behavior}

Since surfaces present, and interact through, electrical and magnetic energies, the electrical characteristics of colloids take on fundamental importance. For example, sick, dead and broken-down cells are attracted to colloids by electromagnetic force, as iron filings are attracted to a magnet. The resulting complexes are carried into the lymph, which recycles what it can, while the rest are carried to the bloodstream to be eliminated. The recycling/breakdown process is carried on by a normal level of fermentation, and it is highly likely that (colloidal) microzymas provide the enzymes as theorized by French scientist and medical doctor- Antione Bechamp.
The surface energies of colloids have powerful effects on physical and chemical activity. It is well known that like charges repel and opposite ones attract, thus surface charges on colloids maintain an energetic system that resists coagulation. Often behaving like enzymes in life processes, certain colloids act as catalysts in chemical reactions. The high surface energies that may accompany them account for the action and sensitivity of colloids in the living system. The electrical potential on the surface of a colloid is known as the zeta potential. Another colloid pioneer, Thomas Riddick, believed zeta potential to be a basic law of nature, or an expression of a universal law-the Law of Balance, or the tension of opposites. Nowhere is this law better represented than in positive vs. negative charge, the fundamental vibrations of elements. Thus, it becomes obvious that zeta potential is basic to life. But each substance, being uniquely configured, will present its own quality of influence (Figure 3).

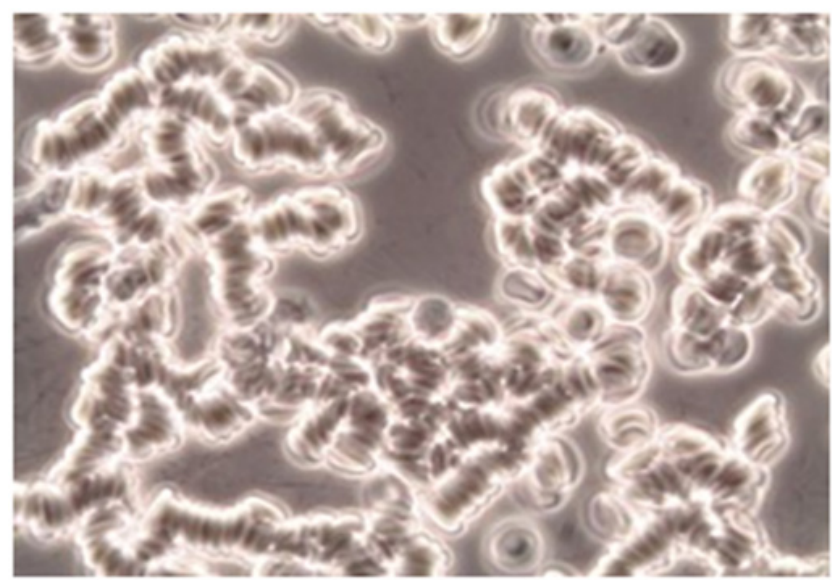

Figure 3 Red blood cell clotting know as Rouleau due to lost surface charge.

\section{Blood clumping (Rouleau)}

These photographs show the blood of a healthy person after eating a large meal with a high fat content. The picture in Figure 3 shows the red blood cells immobile and aggregated due to loss of surface charge or zeta potential. At this point the person felt tired and listless. Figure 4 pictures is the same person's blood 15 minutes after drinking a glass of alkaline water with 5 drops of liquid colloidal alkalizing minerals. ( $\mathrm{pH}$ Miracle's Liquid Lightning Siver DepHense Minerals ${ }^{\mathrm{TM}}$ were used).

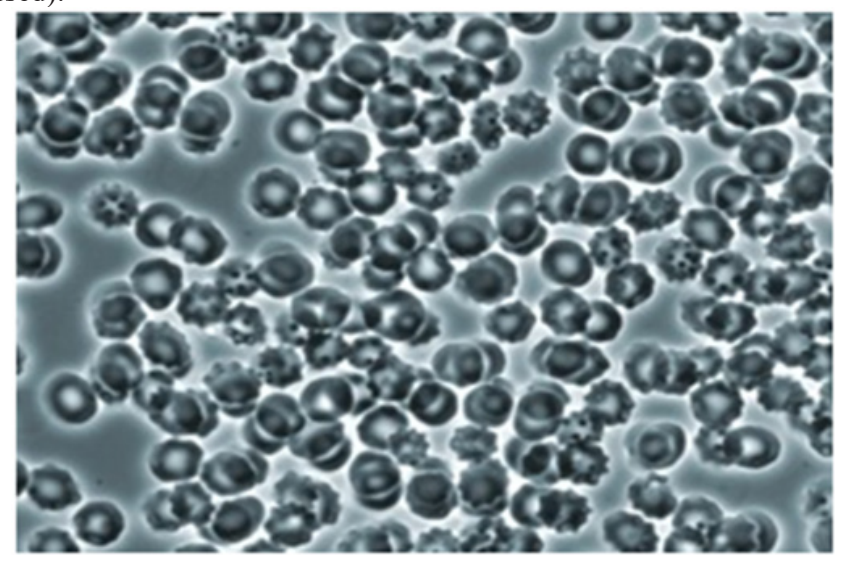

Figure 4 The normal profile of red blood cells is to be separate residing in their own space.

These highly negatively charged particles impart a vibrational energy which breaks the cells apart and restores the zeta potentialwhat I call the negative spark of life. Blood cells are once again 
discrete and highly mobile, regaining their efficiency as carriers of oxygen. The subject reported feeling bright and energetic.

\section{The colloidal computer}

The human body is literally a gigantic liquid crystal! -Robert $\mathrm{O}$. Becker.

We know today that all living organisms are composed of highly structured colloidal systems and that these form the basis of a gigantic colloidal computer. Every cell has an internal colloidal system arranged in patterns to create specific functions. The cells surrounding nerve fibers-glial cells in the brain, Schwann cells in the rest of the body-are made up of high-zeta-potential colloids arranged in a structured matrix. (The reader may be aware that the difference between Einstein's brain and ordinary brains is that his brain had more glial cells).

Dr. Robert O Becker, formerly of the Upstate New York Veterans' Hospital, is considered the world's foremost authority on electrically accelerated healing of tissues. In his book The Body Electric, he describes his discovery of an analog computer-type healing system in the body. (Until he made this discovery, it was thought that the nervous system was a digital information transfer system.) In an analog computer, information is transmitted by voltage levels or by current intensity. Becker found that glial and Schwann cells form the basis of a direct-current, analog healing system for broken bones. The body produces a negative electrical current across the break, which mediates the healing process-by attracting nutrient ions to the area, for one thing.

He also found that glial cells and Schwann cells are semiconductors just like transistors and that there is a direct correspondence between these cells and the acupuncture energy system mapped out by the ancient Chinese. (Despite his brilliance, however, Becker joins other pioneers in being ignored and shunned by establishment savants because his work threatened vested interests and carefully guarded concepts on several fronts. Thus, for one thing, a marvellous development of his-a safe, effective method of regenerative, scar less healing of severe injury-has been denied to the world).

The body has a special mechanism-called, for convenience, the blood-brain barrier-that somehow prevents many substances from entering the brain. It is very likely an electro $\neg$ chemical condition in which the balance of electrolytes in the brain and nervous system is critical. When the electrolyte balance is disturbed and the crystalline structure of this vital liquid changes, unwanted materials may pass the barrier. Since a colloidal system supports intercellular fluid, the same situation may exist here, so that if the colloidal system is compromised, the electrolytes lose their ability to reject unwanted substances.

Dr. Becker has shown that the "permeability" of the barrier is altered by external, pulsed electrical and magnetic fields of extralow frequencies (ELF, or 1 to $100 \mathrm{~Hz}$ ). ELF signals are transmitted by power lines and hundreds of industrial and household products. Everything from hair dryers, TV sets, computers, washing machines, clothes dryers, even electric wrist watches emit these low frequency waves. Dr. Becker believes the health and possibly the existence of the human race is threatened by these signals, as they alter the chemistry of life (an area in which he threatened money interests). Experiments have shown that colloidal dispersion is threatened with collapse by ELF signals when the zeta potential of the colloidal system is low. At this point it takes very little stress to trigger flocculation in the system. This occurs with most colloids at a zeta potential of around 1 millivolt.

\section{Dead zones}

It is possible that the increase in zeta potential achieved by adding high energy colloids and anionic electrolytes to the body will protect us from these harmful energies. As noted, colloids and electrolytes relate to and support one another by their electrical bio-energy. Health is vitally characterized by organized matter that carries a high negative surface charge. Death/disease in the terrain is the neutralization of a colloidal system in an area of the body by the positively charged influence of toxins, residues from acid-forming food, and resulting morbidly evolved microforms and their acids. I refer to such areas as dead zones. They exist in the blood as rouleau, as fused cells in tissues, and in the intercellular fluid itself (see below). Toxins and debris in the system promote loss of negative surface charge and act as mortar to glue cells together, reducing function and efficiency. Given the chance, the body will deal with these areas in an electrical manner somewhat analogous to Dr. Becker's revelations about broken bones. Here, however, the electricity is not a current, but is static. Highly negatively charged colloidal mineral particles will be attracted to these zones, exert their influence and re-establish balance, allowing blood cells to dissociate and opening up cellular communication in tissue. They do this by providing the "negative spark of life."

\section{Intercellular fluid}

A most crucial area for appreciating the significance colloids and their flocculation, is the intercellular fluid, or body humours. As described by OC Gruner the flow of life is maintained in the space between cells, where the living system has enclosed part of the ocean, so to speak. This humeral milieu, or "lake" as Gruner refers to it, has inflow and outflow. The specialized cells of the "tissue sponge work" rely upon this substance that bathes them, maintains $\mathrm{pH}$, brings in nutrients, carries away wastes, and shields them from toxins. The flow moves from the capillaries through the interstitial spaces, then, after exchanges with the cells, back into the lymphatic channels.

\section{Some remarks from Gruner}

"Unless the outlet of the pool is patent and the canaliculi likewise, chemical changes take place in the stagnant fluid. Moreover, the parts beyond cease to receive the materials needed.... Should this movement be arrested in any part of the body, however small be the site, and however short be the time-what we call "disease" begins. ... It may be some time before the signs of obstruction become evident... for the surplus capacity of the "lake" is so great. . . . We have this principle then: there is only one disease. All the 414 (or so) diseases described in textbooks of medicine are fundamentally forms of one and the same disorder.... The problem to be solved in every case of sickness is, "for what reason has the flow of intercellular fluid ceased, and in what site has it ceased?" But the final reason is the presence in the fluid of the "pathogen," whose makeup is chemical emphasis added."

The pathogen is of course not a microform but a toxin. Nutritional deficiency, the other major pathogenic factor, must also be kept in mind.

\section{Colloids and inner light}

In emphasizing the importance of the dynamics of the lake, Gruner describes an "....intangible circulation .....namely the flow of 'vitality', life-force, bio energy, along the neuro-endocrine system, following the physiological processes of absorption, assimilation, and elimination. As long as this flow is unhindered, the individual is in a state of health." It is the extraordinary surface area of the colloidal system which makes it capable of carrying a high level of charge and 
thus vibrational energy, or bio energy. The neuro-endocrine system, the neuro-lymphatic system and the muscular system are all tied together via the meridians identified by the Chinese over 5,000 years ago and used in acupuncture ever since. And the operating principle of acupuncture recalls the yogic understanding of prana (life force) and the chakras. The Law of Balance is again manifest as the yin and yang aspects of life force. Thus, in addition to food, the body ingests, assimilates and excretes subtle vibratory energies, including normally invisible light frequencies.

An interesting aspect is that Gruner sees both the lake and the lymphatics as vital sites of parenteral digestion. In the lymphatics: "There is a real digestion of the entering fluid carried on by the ferments emphasis added supplied by their cell population." And in the intercellular fluid: "The 'lake' is the site of parenteral digestion. This is the real digestion. It is the site of constant building up and tearing down, through enzyme activity." As indicated, poisonous waste products from dietary improprieties find their way into the fluid and interfere with the flow because they cannot be perfectly digested. They are "acid decomposition products," which create a condition of anoxia in the fluid:

Besides this anoxia.... the lack of ferment emphasis added needed to break up the pathological substances itself causes interference with the onward flow.... Failing the breakdown of the materials in question, deposition will occur. As Lumiere pointed out, it is not the chemistry per se that accounts for health, it is the due retention of the colloidal state emphasis added. When flocculation (precipitation, deposition) occurs, the signs of disease appear. ... To sum up, the effects of interference are evident to the clinician in all the manifold forms which disease assumes.... Their real significance is appreciated in terms of biocolloidology.

What structure could be responsible for most or all of the critical fermentations and anabolic enzyme activity carried on in this inner sanctum? Our fundamental anatomical elements, the colloidal microzymas of the brilliant Antoine Bechamp? It is the electrical, or bioenergetic nature of the fluid that supports the flow, and it is primarily a colloidal system, in concert with (molecular) electrolytes, that constitutes its electrical nature. A fascinating point to be made with respect to anabolic activity is that Gruner believes the lake to be the birthplace of cells, which must also be the work of the tireless and indestructible microzymas.

With regard to the presence of light in living systems, recent discoveries indicate that colloids play a critical role in the vital function of intercellular communication. Discoveries by Fritz Albert Popp of Germany indicate that the DNA molecule transmits its blueprint information to other cells by means of encoded bursts of ultraviolet laser light. The optical pathways for this information are "light pipes" consisting of highly structured cellular water (Fusion, Sept./Oct. 1985). Popp indicates that the structure of cellular water is created by minute quantities of highly charged colloidal minerals.

\section{Applications in health}

In the billions of cells comprising tissues and organs, energy is obtained from certain metals, among them iron, iodine, manganese and copper. There are some 32 minerals in the body, with traces of at least as many others. Colloidal nutritional chemistry is the science which converts those elements into particles so minute they can be utilized directly by cells and the intercellular fluid.

Ideally, a nutrient should be administered in such a form that its essential constituent will travel through the body until it reaches the part where it is required, and that it arrives at that organ or tissue in such a state as to be used to the greatest advantage. To administer a substance in chemical form because it has been isolated from brain matter, for example, may be to misunderstand the chemical and physical changes which take place in assimilation, and thus to supply the material in a form from which it has to be converted. The fact that this conversion occurs shows how marvellous an alchemist the human organism is; but it is no reason for the administration of agents in less than ideal form, especially when the physiology is weakened. The treatment of a disease condition by the administration of various compounds is much more completely understood when it is realized that the reactions deal largely with colloidal materials and systems. Nutritional and remedial treatments become most efficient when they can be based entirely upon this principle or can include it, eliminating or minimizing further disturbance to the system.

Before the 1940s, even though certain colloids had seen substantial success-colloidal silver for example-the full health potential of colloids in general had not been demonstrated. This is due to a number of factors, of which the two most important are: (1) the difficulty of studying the special properties of substances in the colloidal state with the technology at hand in the first three decades of the 20th century; (2) failure of early investigators to realize the necessity of stabilizing the remedial colloid in terms of rendering it is osmotic with the blood serum, thus preventing precipitation. Then, in 1941 and later, antibiotics and other "miracle drugs" came on the scene, and mainstream interest turned away from the great potential of colloids. Thus, their potential has waited to outlive the devastation of health by drugs and has become more fully realized only in the last decade.

\section{Stabilization}

It is now quite evident that the application of colloidal sols to the disease condition of the human body is distinctly encouraging. As suggested, a supply of improperly prepared and unstable colloidal systems has been one of the most serious sources of drawbacks and discouragement. In sols, the presence of very small quantities of certain salts, the ions or ultimate particles of which have an electrical charge opposite to that of the active colloid, will coagulate the latter unless it is protected by some means. In this case, coagulation or precipitation can occur only when the protective agent has been destroyed or removed.

In the 1970s, upon recognition of the colloidal nature of the chief body fluids, German investigators saw the enormous possibilities for the application of colloidal disinfectants and medicines. Subsequently (1980s), several colloidal products were placed on the market and their therapeutic properties carefully and vigorously "hyped." Most of these systems rapidly deteriorated in value, however. Some were so unstable they contained no active colloid at the time of use, and others, not being is osmotic with body fluids, coagulated immediately upon administration.

On the other hand, if a substance is converted to the colloidal state in the presence of certain other colloids and of certain salts, the activity of the desired colloid will not be impaired and the system will be quite stable, mixing easily with blood or other body fluids without being rendered inactive by them. A potential drawback is that, while the additional colloids and salts enable use under otherwise impossible circumstances, the action of the protective agents becomes a factor. Usually it appears to be negligible, but occasionally it has been observed to be of considerable importance.

\section{Practicality}

A great advantage which colloidal sols of elements possess over compounds is the facility with which their action may be studied. If 
a salt or other compound is administered, there is always the chance of it undergoing hydrolysis or ionization in the alimentary canal or bloodstream, thereby setting up a complex situation in which elements other than the one under investigation become involved. For example, iron may be administrated in the form of a carbonate which is converted in the stomach into chloride and this, on dilution, is hydrolyzed so that eventually there are formed both hydroxide and chloride of iron. If the iron were administered as a colloidal element, these complications would be avoided and the investigator would be much more certain in drawing conclusions.

Also, an element in the ionized state is always associated with its corresponding ion(s). Thus, ionized potassium chloride separates into potassium ions and chlorine ions, and the net charge of the system is neutralized. When the element is administered in the colloidal state, however, it is introduced alone as an active agent, the charge in the particles is quite definite, and activity is correspondingly great. Also, the extreme toxicity of many combined or non-colloidal elements is avoided when administered in the form of colloidal sols, while usefulness is brought to the fore. The remarkable fact that colloidal silver and iodine do not stain the skin, whereas pharmaceutical preparations of silver and iodine do so strongly, is a further indication of the striking difference between colloidal sols and ordinary solutions.

The effect of administering elements in a colloidal state to persons suffering from certain symptomologies is extremely interesting, partly because of the progress of the recovery, and partly because of the absence of complications such as occur when the same element is administered in another form. Consequently, there is much truth in the statement that a substance to be fully efficient must be in a colloidal state, or very easily convertible to it in the body of the subject.

\section{Power}

Many metallic colloids serve as powerful catalysts. For example, colloidal silver, titanium, gold, platinum, rhodium, iridium, osmium and palladium are effective catalysts in the despecialization and respecialization of cells. I have observed that these highly negatively charged metal sols can cause red or white blood cells to dedifferentiate to their embryonic state and then redifferentiate into cells needed due to emotional or physical stresses, e.g. bone, muscle, nerve or skin cells. This phenomenon was seen in a patient with third degree burns on his hands and face. As silver and titanium metal sols were administered topically, new skin grew rapidly through the respecialization of blood cells. Even where there were skin grafts, new skin was created underneath them, where the metal sols were administered. In The Body Electric, Dr. Becker also reports observations of the healing power of the negative charge.

The electrically charged particles of some metal sols have activity and catalytic power so great as to be barely conceivable. They can induce chemical reactions which would otherwise require conditions unattainable in the human subject. Dr. Hirschberg found that they cause strong chemical actions "out of all proportion with the quantity of matter brought to bear." Marked catalytic properties are evident when only 0.0000002 grain of a platinum sol is present, for example. This intense power of promoting reactions and of being themselves left free at the end of the reaction results in very small quantities of metal sols being capable of effecting changes highly disproportionate to the amount of sol present. Changes which are extremely complex or which would otherwise require a long time if relying on a series of successive reactions may be readily effected by the presence of an elemental sol. These considerations throw light on the importance of trace elements in nutrition. Metal sols have the further therapeutic advantage of acting most rapidly in slightly alkaline solutions, so that when properly prepared they are not affected adversely by normal blood.

Of interest is the fact that substances (e.g., mercuric chloride or arsenic acid) that block catalysis by metallic colloids have a like effect on biological systems-that is, they block biochemical reactions and hence can be lethal.

\section{Conclusion}

The use of specific colloids in health is not a universal cure-all, but just as the administration of extracts or isolates marks an advantage in many cases over the use of the cruder source, so the employment of colloidal elements marks a clear step forward in many cases over traditional supplements or remedies. The ability to energize blood and body fluids alone offers major support to health and a means of addressing a fundamental physiological dysfunction. From this standpoint, the positive effect of colloidal supplements on a host of symptomologies could be anticipated, and has been observed clinically.

\section{Acknowledgments}

None.

\section{Conflicts of interest}

None.

\section{Funding}

None.

\section{References}

1. Voyutsky S (1978) Colloid Chemistry. Moscow: Mir Publishers, Moscow, Russia.

2. Webster DA, Spadaro JA, Becker RO, Cramer S (1981) Silver anode treatment of chronic osteomyelitis. Clinical Orthopedic and Related Research 161: 104-114.

3. Alexander J (1924) Colloid Chemistry. Van Nostrand Co., New York, USA.

4. Becker RO, Selden G (1985) The Body Electric. Electromagnetism and the Foundation of Life. QuillAVilliam Morrow, New York, USA.

5. Becker RO and Spadaro JA (1978) Treatment of orthopaedic infections with electrically generated silver ions. A preliminary report. J Bone Joint Surg Am 60(7): 871-881.

6. Becker RO, Spadaro JA (1979) Experience with low current silver electrode treatment of nonunion. In: Grighton CT, Black, Jonathan B, et al. (Eds.), Electrical Properties of Bone and Cartilage. Grune and Stratton, New York, USA, pp. 631-638.

7. Freundlich H (1922) Colloid and Capillary Chemistry. EP Dutton, New York, USA.

8. Freundlich H (1925) The Elements of Colloidal Chemistry. Methuen Publishing, London, UK

9. Goddard ED (1985) Colloid. The World Book Encyclopedia, Chicago, USA.

10. Gruner MD and Cameron O (1947) An Interpretation of Cancer. Health Research, Washington, USA.

11. Higher Education Library Publishers (H.E.L.P.). Colloidal Silver-A closer look. H.E.L.P.fulNews 9 p. 11

12. Hirschberg, Leonard Keene. Electrical Colloids, from an article out of Johns Hopkins University Hospital. 
13. Searle AB (1919) The Use of Colloids in Health and Disease. EP Dutton \& Co., New York, USA.

14. RO Young (1999) Sick and Tired, Reclaim Your Inner Terrain. Woodland Publishing, Orem, Utah.
15. RO Young, SR Young (2010) The pH Miracle revised and updated. Hachette Publishing and Grand Central Publishing, New York, USA. 